\title{
A Rocking-Chair Proton Battery with Conducting Redox Polymer Active Materials and a Protic Ionic Liquid Electrolyte
}

Huan Wang, ${ }^{\text {a }}$ Rikard Emanuelsson, ${ }^{\text {a* }}$ Christoffer Karlsson, ${ }^{\mathrm{b}}$ Patric Jannasch, ${ }^{\mathrm{b}}$ Maria Strømme, ${ }^{\mathrm{a}}$ and Martin Sjödin. ${ }^{a *}$

a, Nanotechnology and Functional Materials, Department of Materials Science and Engineering, The Ångström Laboratory, Uppsala University, Box 35, SE-751 03, Uppsala, Sweden

b, Centre for Analysis and Synthesis, Department of Chemistry, Lund University, P. O. Box 124, SE-221 00

Lund, Sweden

H. Wang, Dr. R. Emanuelsson, Prof. M. Strømme, Prof. M. Sjödin

Nanotechnology and Functional Materials, Department of Materials Science and Engineering

The Ångström Laboratory, Uppsala University, Box 35, Uppsala SE-751 21, Sweden

*E-mail: martin.sjodin@angstrom.uu.se

*E-mail: rikard.emanuelsson@angstrom.uu.se

Dr. C. Karlsson, Prof. P. Jannasch

Centre for Analysis and Synthesis, Department of Chemistry, Lund University, Box 124, 22100 Lund,

Sweden

\section{Abstract}

Rechargeable batteries that use redox-active organic compounds are currently considered as an energy storage technology for the future. Conducting redox polymers (CRPs) are organic materials being both electronically conducting and resilient to dissolution. While insolubility is an advantageous property for active battery materials, it complicates the processing necessary for fabricating electrodes, including electrode formulation and layer formation. Here we employ a postdeposition electro-polymerization (PDP) method, which allows for solution-processing to be used for electrode layer formation. The polymerization conditions are optimized and the underlying mechanism is studied with the final aim to produce high performance CRPs as energy storage materials. We show that quinizarin $(\mathrm{Qz})$ and naphthoquinone (NQ) based CRPs can reach their theoretical capacity thorough optimization of the polymerization conditions. Combining the two CRPs, with the Qz-CRP as cathode and the NQ-CRP as anode, and a protic ionic liquid electrolyte, yields a $0.8 \mathrm{~V}$ proton rocking-chair battery. The conducting additive-free all-organic proton battery exhibits a capacity of $62 \mathrm{mAh} / \mathrm{g}$ and a capacity retention of $80 \%$ after 500 cycles using rapid potentiostatic charging and galvanostatic discharge at $4.5 \mathrm{C}$. 


\section{Introduction}

The development of large-scale power systems such as electric vehicles and smart grids escalates the demand for energy storage technologies, such as batteries. Most batteries, however, depend on unsustainable inorganic materials and suffer from environmental issues and high $\mathrm{CO}_{2}$ footprint. ${ }^{[1,2]}$ Significant research focus has therefore been put on replacing inorganic energy storage materials used in traditional lithium ion batteries with sustainable, earth abundant, low $\mathrm{CO}_{2}$-footprint, and cheap organic materials (containing $\mathrm{C}, \mathrm{H}, \mathrm{O}, \mathrm{N}$ ) that, in addition, would provide simplified end-of-use treatments. ${ }^{[3,4]}$

The quinones, as some of the simplest carbonyl compounds, are particularly attractive as charge storage components due to their high specific capacities and reversible and fast 2-electron (2e) redox reactions. ${ }^{[5-7]}$ Moreover, electron-withdrawing and electron-donating substituents as well as fused aromatic rings can effectively tune the quinone redox potential by altering the electron density of the quinone core. ${ }^{[8,9]}$ In addition, the versatile quinone redox chemistry is compatible with several different cycling cations, including alkali metal cations (e.g. $\left.\mathrm{Li}^{+}, \mathrm{Na}^{+}\right)$, organic ammonium cations and protons, as well as with different solvents. ${ }^{[10-12]}$

Utilizing protons as cycling ions is particularly interesting as the proton is light, abundant and has the highest diffusion coefficient known to date. ${ }^{[13-15]}$ There has been significant progress in the development of proton batteries during the last few years with respect to voltage output, discharge capacity as well as stability. Yao's group used a pyrene-4,5,9,10-tetraone as anode together with $\mathrm{PbO}_{2}$ as cathode, making a $1.2 \mathrm{~V}$ aqueous proton battery with a capacity retention of $96 \%$ after 1500 cycles. ${ }^{[16]}$ In our previous report, naphthoquinone (NQ) and hydroquinone were functionalized onto a conducting polymer, making a conducting additive-free $0.4 \mathrm{~V}$ aqueous proton battery, showing $85 \%$ capacity retention after 500 cycles. ${ }^{[17]}$ Honma's group instead combined an anthraquinone anode with a tetrachlorohydroquinone cathode in an aqueous battery that extended the voltage output to $0.6 \mathrm{~V} .^{[18]}$ Using a symmetric 2,3-dimethyl-quinizarin as both cathode and anode Aziz's group presented a battery with a voltage output $(1.16 \mathrm{~V})$ that closely matched the stability window of water $(1.23 \mathrm{~V}) .^{[19]}$ 
The capacity retention was, however, rather limited, with $47 \%$ of the initial capacity remaining after 100 cycles. The limited stability can be traced to the water solvent as several reports show that in the oxidized state quinone can react with water through Michael addition. ${ }^{[19-23]}$ By replacing the water solvent it should therefore be possible to increase the stability of proton batteries by suppressing the Michael addition reaction. This could be done in organic protic electrolytes ${ }^{[24]}$ or using nonstoichiometric protic ionic liquids, ${ }^{[25]}$ which also could allow a potential window over $1.23 \mathrm{~V} .{ }^{[26-28]}$ We have previously shown that the use of non-aqueous solvents significantly extends the possibility to tune quinone redox potentials by substitution, since in aqueous solution, specific interactions with water molecules counteract the effect of the substituents. ${ }^{[29]}$ Finally, protic ionic liquids may provide a solution for redox-active materials that show poor wettability in water electrolyte. One type of protic ionic liquids is composed of positively charged, protonated nitrogen heterocycles and a suitable charge compensating anion. In non-stoichiometric protic ionic liquids, only a portion of the nitrogen heterocycle moieties is protonated and hence the electrolyte can act as both proton acceptor and proton donor and can thus sustain proton coupled redox reactions. The acidity and relative fraction of the protonated (acid) and the unprotonated (base) heterocycle moieties can be used to tune both the kinetics and the energetics of proton coupled redox reactions. ${ }^{[25]}$

Herein, a 1-methyl-1,2,4-triazole (MeTri) based ionic liquid $\left(\mathrm{pK}_{\mathrm{a}}=3.2\right)$ was used as electrolyte together with conducting redox polymer (CRP) electrode materials. Capacity-carrying quinizarin (1,4-dihydroxyanthraquinone, Qz) and NQ pendants covalently attached onto a conducting polymer backbone were used as cathode and anode, respectively. The conducting polymer backbone provides electron transport pathways for the pendants' redox reactions and also prevents the dissolution of pendants. A conducting additive-free all-organic proton rocking-chair battery was thereby fabricated (Scheme 1). 

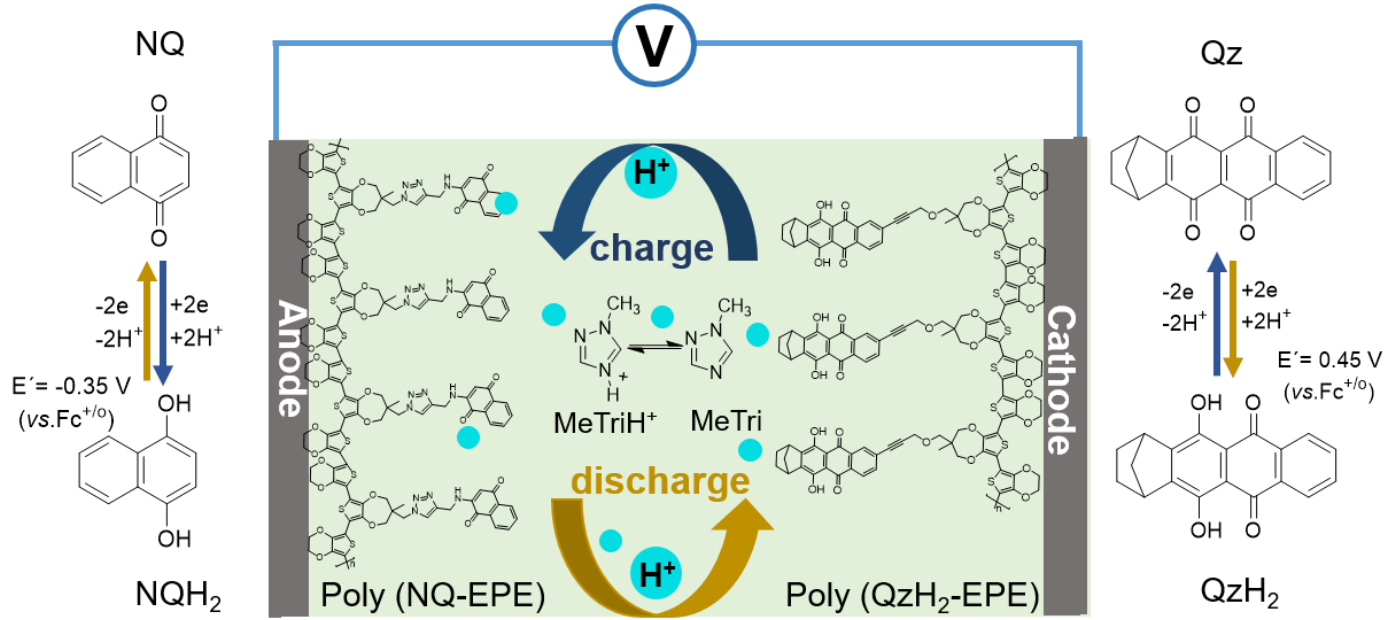

Scheme 1. Schematic illustration of the working principle of a NQ-Qz rocking-chair proton battery.

\section{Results and discussion}

\subsection{Post-deposition electro-polymerization}

The prepared trimer film was polymerized using the PDP method, ${ }^{[17]}$ which allows the full utilization of starting materials as opposed to traditional polymerization from a monomer solution, where most starting materials remain unreacted. In this method a layer of the repeating unit precursor is immersed into an electrolyte solution (the polymerization solution), where the layer is oxidized by oxidants in the electrolyte solution or by electrochemically applying a sufficiently positive potential to the layer. ${ }^{[17]}$ An important prerequisite for this method to work is that the initial precursor-layer does not dissolve in the polymerization solution. This can be achieved by the choice of polymerization solution as well as by using precursors that interact strongly with each other. Here, we use thiophene-based trimer structures with extended $\pi$-systems that are likely to interact by $\pi-\pi$ stacking. The utilization of trimeric structures as precursors also ensures a lower oxidation (polymerization) potential compared to monomeric analogues (above $1 \mathrm{~V} v s$. $\mathrm{Fc}^{+/ 0}$ ) allowing for milder polymerization conditions to be used. In contrast to monomeric analogues the trimeric precursors also exhibit electronic conductivity upon oxidation, allowing for electronic communication through the trimer layer. 

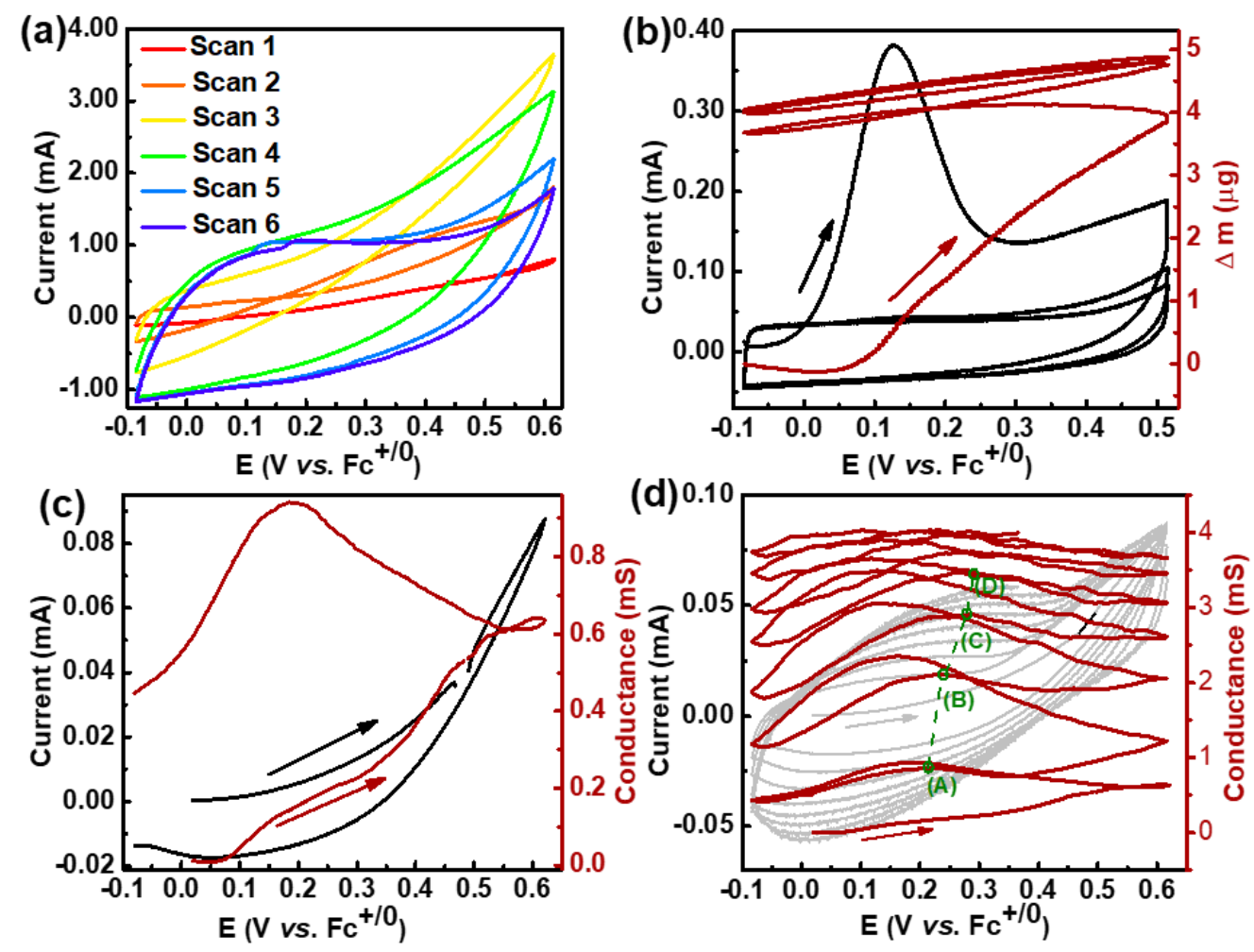

Figure 1. (a) Cyclic voltammograms recorded during polymerization of $0.5 \mathrm{mg}$ NQ-EPE deposited on a glassy carbon plate at $20 \mathrm{mV} / \mathrm{s}$, (b) Cyclic voltammograms (black) and corresponding mass change (red brown) during the polymerization of $10 \mu \mathrm{g}$ NQ-EPE on an EQCM Au-electrode at 20 $\mathrm{mV} / \mathrm{s}$. The arrows indicate the first anodic scan. Conductance (red brown) and cyclic voltammograms (black) of (c) the first polymerization scan, and (d) first seven polymerization scans of $10 \mu \mathrm{g}$ NQEPE on IDA Au-electrode at $50 \mathrm{mV} / \mathrm{s}$. The points A, B, C, and D represent the potentials where the conductance reaches its maximum value during anodic scan. The electrolyte in the above experiments is $0.1 \mathrm{M}$ MeTriHTFSI/MeCN/H$/ \mathrm{H}_{2} \mathrm{O}$ (Vol MeCN: 67\%).

We use thiophene-based EPE as repeating unit while NQ and Qz, covalently attached to the central ProDOT unit, are acting as capacity-carrying components for the anode and cathode, respectively (Scheme 2). The brown NQ-EPE $100 \mathrm{mg} / \mathrm{mL}$ trimer solution with 10wt\% PVDF (with respect to NQEPE) was drop-cast onto a glassy carbon current collector and vacuum-dried, forming a dark brown film. The glassy carbon electrode was then transferred to $0.1 \mathrm{M}$ MeTriHTFSI/MeCN/ $\mathrm{H}_{2} \mathrm{O}(\mathrm{Vol} \mathrm{MeCN}$ : $67 \%$ ). (Figure S14). No dissolution of the layer was observed prior to polymerization, confirming 
that the trimeric precursors have limited solubility in $0.1 \mathrm{M}$ MeTriHTFSI/MeCN/ $/ \mathrm{H}_{2} \mathrm{O}(\mathrm{Vol} \mathrm{MeCN}$ : $67 \%$ ) electrolyte. $\mathrm{CV}$ between $-0.1 \mathrm{~V}$ and $0.6 \mathrm{~V}\left(v s . \mathrm{Fc}^{+/ 0}\right)$, was then used to polymerize the trimer layer in the above electrolyte during which the dark brown NQ-EPE trimer layer turned into black poly (NQ-EPE) film. Photographs of the layer in each step during PDP method are shown in Figure $\mathrm{S} 15$. In the first anodic CV scan the current rises from $0.2 \mathrm{~V}\left(v s . \mathrm{Fc}^{+/ 0}\right)$ (Figure 1a), which results from the oxidation of neutral trimer. Oxidation of the trimer signifies the formation of trimer radical cations which can attack and couple to another trimer radical and form a hexamer. The hexamer can further be oxidized and couple to another radical and the polymer grows. The observed trimer oxidation potential is much lower than that of the monomer $\left(1.1 \mathrm{~V} v s . \mathrm{Fc}^{+/ 0}\right),{ }^{[32]}$ which is attributed to the extended aromatic system in the trimer. ${ }^{[33]}$ In the following three scans, polymerization continues with observed irreversible oxidation currents above $0.2 \mathrm{~V}\left(v s . \mathrm{Fc}^{+/ 0}\right)$. During polymerization a reversible, seemingly capacitive current, resulting from doping (oxidation) and dedoping (reduction) of the polymer backbone, builds up at potentials below $0.2 \mathrm{~V}\left(v s . \mathrm{Fc}^{+/ 0}\right)$. The polymerization is completed in five scans and only the rectangular-shaped capacitive current is observed between -0.1 $\mathrm{V}$ and $0.6 \mathrm{~V}\left(v s . \mathrm{Fc}^{+/ 0}\right)$. The polymerization of $\mathrm{QzH}_{2}$-EPE shows similar behavior as NQ-EPE except that the Qz/QzH 2 redox peak centered at $0.4 \mathrm{~V}$ is observed and the peak current also builds up upon polymerization (Figure S16). Infrared (IR) spectroscopy shows that the characteristic vibrational peaks of quinone and trimer backbone were well-preserved after polymerization (Figure S26). The length of the resulting polymers was estimated from the charge consumed during polymerization (Figure S21 and S22). For poly (NQ-EPE) and poly (QzH2-EPE) the average polymer lengths were calculated to be 14 and 9 thiophene units, respectively. The polymer lengths obtained from the PDP method was thus comparable to what is commonly found for polymers derived from traditional chemical and electrochemical polymerization methods. ${ }^{\left[{ }^{[3,35]}\right.}$ In situ EQCM measurement revealed an extensive mass increase above $0.07 \mathrm{~V}\left(v s . \mathrm{Fc}^{+/ 0}\right)$ during NQ-EPE polymerization (Figure 1b). Both polymerization-induced solvent uptake and doping-induced uptake of charge-balancing TFSI anions are associated with mass increase. Assuming that the uptake of TFSI is reversibly expelled during the 
following cathodic scan the solvent uptake can be estimated by the net mass change after the complete first cycle. Such estimation suggests $30 \mathrm{wt} \%$ and $49 \mathrm{wt} \%$ solvent uptake for NQ-EPE and QzH 2 -EPE, respectively (Figure S17). The PDP-process is thus associated with a substantial swelling during polymerization.

In situ conductance measurements during polymerization show that the conductance starts to increase from $0.1 \mathrm{~V}\left(v s . \mathrm{Fc}^{+/ 0}\right)$ during the first anodic scan (Figure 1c). Polymerization of trimer produces polymer chains with increased conductance compared to trimer as a result. The conductance increases steadily even after scan reversal until $0.2 \mathrm{~V}\left(v s . \mathrm{Fc}^{+/ 0}\right)$ due to the continuous polymerization at sufficiently high potentials, as confirmed by the positive currents observed during cathodic polarization. During the anodic sweep in scan 2 the conductance initially increases, as the obtained polymer is doped. However, the conductance reaches a maximum value of $0.9 \mathrm{mS}$ at $0.21 \mathrm{~V}\left(v s . \mathrm{Fc}^{+/ 0}\right)$ and decreases as the potential increases further (Figure 1d). The potential where the conductance reaches its maximum value is denoted as $\mathrm{E}_{\text {max }}^{\mathrm{G}}$. As the polymerization proceeds in consecutive scans the overall conductance increases, $\mathrm{E}_{\text {max }}^{\mathrm{G}}$ shifts towards higher potentials and the conductance peak becomes less and less pronounced (Figure S18). These features are well accounted for by the conversion of trimeric units to successively longer chains. When the chains are short, charge carriers are localized and electron transport is dominated by redox hopping between localized states. The conductance maximizes when half of the states are populated, i.e. at the average formal potential of the oligomers. ${ }^{[36-38]}$ As the polymer length increases, the increased delocalization of charges increases the interaction between successively induced charges and eventually leads to the continuous doping over an extended potential region observed for most conducting polymers. The broadening of the doping potential window is manifested in the appearance of a conductance plateau seen in the seventh scan (Figure S18f). Conductance monitoring during $\mathrm{QzH}_{2}$-EPE polymerization shows similar behavior as in NQ-EPE: the conductance builds up during polymerization and a conductance maximum is observed. However, the conductance peak does not evolve into a conductance plateau 
upon repeated cycling, indicating that the PDP process results in shorter chains of poly $\left(\mathrm{QzH}_{2}-\mathrm{EPE}\right)$ than that of poly (NQ-EPE) in accordance with the polymer length estimated above.

\subsection{PDP solution optimization}

Traditional electropolymerization from a monomer solution relies on the encounter of two radical cations formed upon oxidation in the vicinity of the electrode surface, and their coupling. The polymer length is largely determined by the polymer solubility as chain propagation is terminated by precipitation. ${ }^{[39]}$ In the PDP method, however, the neutral trimers should not dissolve in the polymerization solution $0.1 \mathrm{M}$ MeTriHTFSI/MeCN/ $\mathrm{H}_{2} \mathrm{O}$ (Vol MeCN: 67\%), which is a prerequisite for the method to work. One might therefore expect that chain propagation should be impossible since the termination condition is already met from start. We attribute the successful PDP above to partial dissolution of the intermediate radical cation formed upon oxidation (Scheme 2, stage ii). This would allow the encounter and coupling of trimer radicals to form longer chains. There should hence be a balance between favorable dissolution of the intermediate that allows for chain propagation, and detrimental dissolution of the neutral trimer causing loss of material.
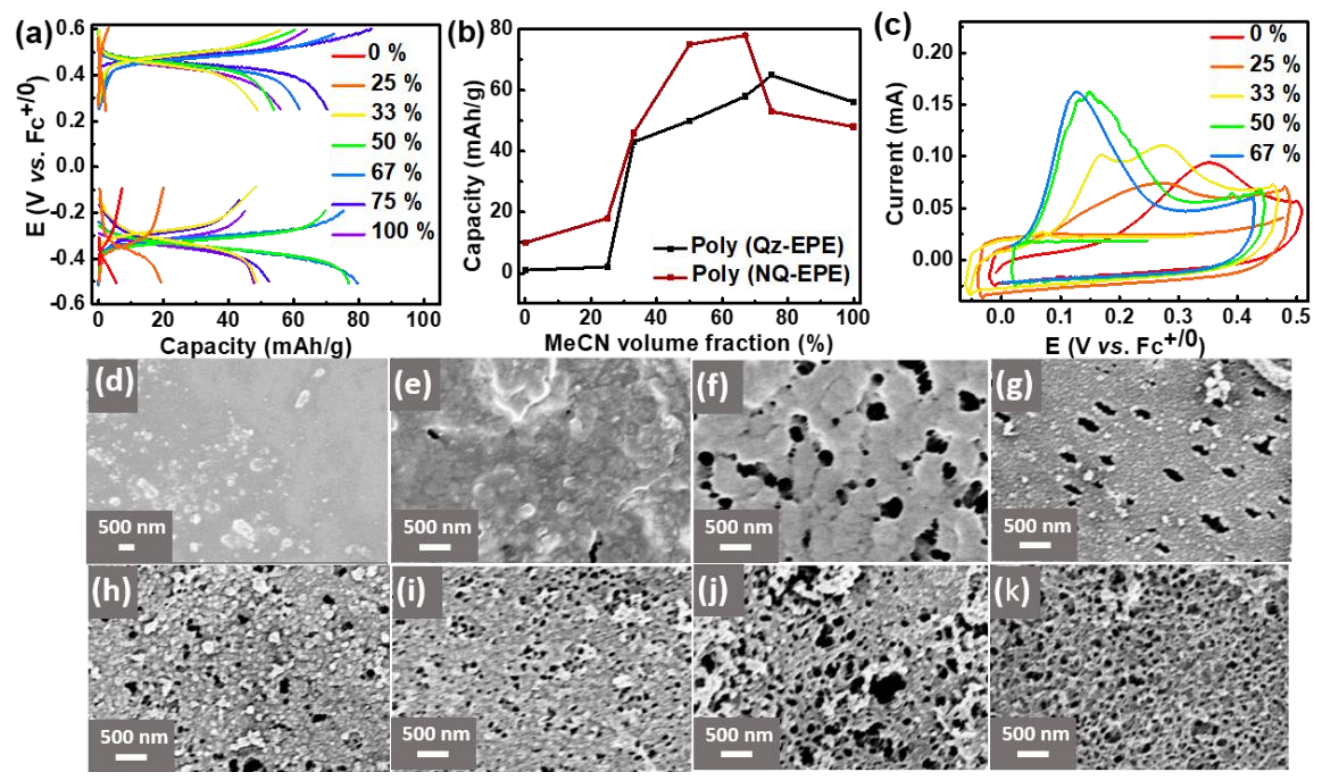

Figure 2. (a) Galvanostatic charge-discharge curves of $0.1 \mathrm{mg}$ poly ( $\left.\mathrm{QzH}_{2}-\mathrm{EPE}\right)$ (upper) and $0.1 \mathrm{mg}$ poly (NQ-EPE) (bottom) tested in a three-electrolyte setup in MeTriHTFSI electrolyte at a current 
density of $0.3 \mathrm{~A} / \mathrm{g}$. Different colors represent polymers polymerized in $0.1 \mathrm{M}$ MeTriHTFSI/MeCN/H${ }_{2} \mathrm{O}$ solution with different $\mathrm{MeCN}$ volume fractions. (b) Corresponding discharge capacity of the two polymers as a function of $\mathrm{MeCN}$ volume fraction in the polymerization solution. (c) Polymerization cyclic voltammograms of $10 \mu \mathrm{g}$ NQ-EPE at $8 \mathrm{mV} / \mathrm{s}$ in $0.1 \mathrm{M}$ MeTriHTFSI/MeCN/H $\mathrm{H}_{2} \mathrm{O}$ with different $\mathrm{MeCN}$ volume fractions. SEM micrographs of NQ-EPE trimer film (d) and poly (NQ-EPE) polymerized in (e) $0 \%$, (f) 25\%, (g) 33\%, (h) 50\%, (i) 67\%, (j) $75 \%$, (k) $100 \% \mathrm{MeCN}$ volume fraction in $0.1 \mathrm{M} \mathrm{MeTriHTFSI} / \mathrm{MeCN} / \mathrm{H}_{2} \mathrm{O}$. All experiments were conducted using a glassy carbon current collector.

To investigate the balance between the favorable dissolution for chain propagation and detrimental dissolution of trimer loss, a series of experiments where the solvent was systematically varied were conducted. The two neutral trimers, $\mathrm{QzH}_{2}$-EPE and NQ-EPE, both dissolve in pure MeCN while neither dissolve in $\mathrm{H}_{2} \mathrm{O}$. A series of polymerization solutions of $0.1 \mathrm{M}$ MeTriHTFSI/MeCN/ $\mathrm{H}_{2} \mathrm{O}$ with different $\mathrm{MeCN}$ volume fractions were used and the resulting polymers were characterized in MeTriHTFSI. Figure 2(a) shows the charge-discharge profiles of the resulting poly (QzH $2-E P E)$ (upper curves) and poly (NQ-EPE) (lower curves) in MeTriHTFSI electrolyte. Poly (QzH2-EPE) exhibited a discharge plateau centered at $0.45 \mathrm{~V}\left(v s . \mathrm{Fc}^{+/ 0}\right)$ which is attributed to Qz/QzH 2 redox conversion (Scheme 1) while the discharge plateau of poly (NQ-EPE) was centered at $-0.35 \mathrm{~V}$ ( $v s$. $\mathrm{Fc}^{+/ 0}$ ) which is attributed to $\mathrm{NQ} / \mathrm{NQH}_{2}$ redox conversion (Scheme 1). The discharge capacity of the two polymers shows a clear dependence on $\mathrm{MeCN}$ volume fraction in the polymerization solution (Figure $2 b$ ) with the capacity reaching the maximum at $67 \%$ and $75 \%$ for poly (NQ-EPE) and poly $\left(\mathrm{QzH}_{2}-\mathrm{EPE}\right)$, respectively. We attribute the decrease in capacity observed at higher $\mathrm{MeCN}$ concentrations to dissolution loss of the neutral state trimer, which was already observed prior to polymerization in these concentrations. For the increase in capacity with increased $\mathrm{MeCN}$ content at low $\mathrm{MeCN}$ concentrations we hypothesize that this is related to the increased rearrangement of the radical intermediate that comes with the increased solubility in the electrolyte. 


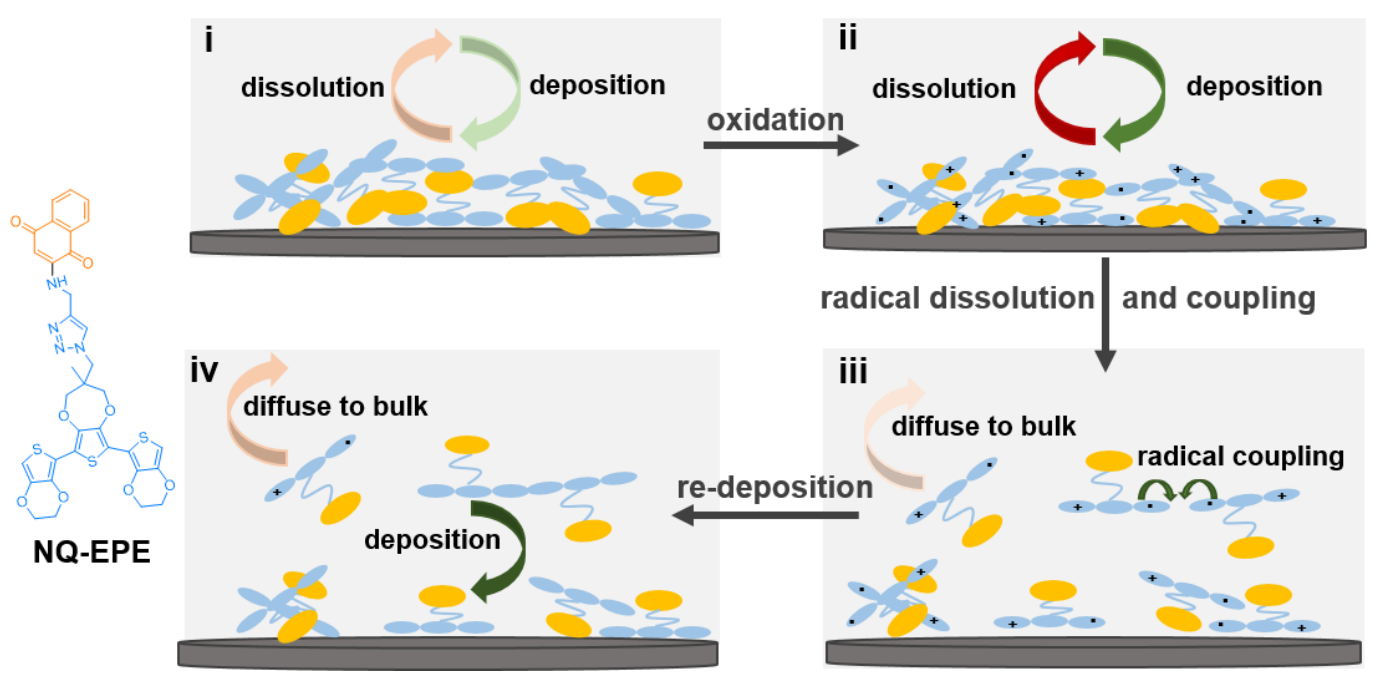

Scheme 2. PDP mechanism (i) glassy carbon with insoluble neutral state trimer film in the polymerization solution, (ii) formation of soluble trimer radical cation upon neutral state trimer oxidation, (iii) competition between trimer radical coupling and diffusion to bulk solution, (iv) oligomer re-deposition.

To test the hypothesis of the rearrangement of radical intermediate, the resulting polymers were further examined. Figure $2 \mathrm{~d}$ shows that prior to polymerization the NQ-EPE trimer film is solid and smooth with almost no microstructure. Polymerization of NQ-EPE caused significant morphological changes indicating that the trimers indeed do rearrange during the polymerization process. In addition, the polymers become increasingly rough and porous with the increased $\mathrm{MeCN}$ fraction in the polymerization solution, indicating that $\mathrm{MeCN}$ facilitated the rearrangements of radical intermediate. With $0 \% \mathrm{MeCN}$ in the polymerization solution the resulting polymer film shows an aggregated structure with a rough, but yet non-porous surface (Figure $2 \mathrm{e}$ ). Using $25 \%$ to $33 \% \mathrm{MeCN}$, the resulting polymer layers become perforated with pore widths on the order of a few hundred nanometers (Figure $2 \mathrm{f}-\mathrm{g}$ ). As the $\mathrm{MeCN}$ volume fraction increases even further $(50 \%$ and $67 \%)$ the polymers consist of $100 \mathrm{~nm}$ nanoparticles with numerous pores of the same size. With even higher $\mathrm{MeCN}$ volume fractions the polymer layer transforms to a highly porous network of $\sim 60 \mathrm{~nm}$ thick interconnected nanowires (Figure $2 \mathrm{j}$ and $2 \mathrm{k}$ ). 
Polymerization of $\mathrm{QzH}_{2}$-EPE also caused morphological changes to the layer that was affected by the $\mathrm{MeCN}$ content in the polymerization solution. However, a MeCN volume fraction of more than $33 \%$ was needed to induce any significant morphology changes as polymerizations using lower MeCN contents gave morphologies that are inseparable from the initial trimer layer (Figure S20). When the $\mathrm{MeCN}$ volume fraction was higher than $33 \%$, polymerization gave a more roughened polymer surface than that of $\mathrm{QzH}_{2}$-EPE trimer. With $\mathrm{MeCN}$ concentrations above 67\% pores start to form during polymerization and above $75 \%$, where the neutral trimer dissolves, the resulting poly $\left(\mathrm{QzH}_{2}-\mathrm{EPE}\right)$ exhibited a porous, nanoparticle-containing structure.

The MeCN content also affects the polymer length: in $0 \% \mathrm{MeCN}$ the average poly (NQ-EPE) was 6 thiophene units while the average polymer length was 14 thiophene units in 67\% MeCN (Table S1). The lower rearrangement ability of $\mathrm{QzH}_{2}-\mathrm{EPE}$ was also reflected in shorter polymer length and we estimate the average polymer length to be 4 and 9 thiophene units for poly ( $\left(\mathrm{QzH}_{2}-\mathrm{EPE}\right)$ formed with $0 \% \mathrm{MeCN}$ and $75 \% \mathrm{MeCN}$ in the polymerization solution, respectively (Table S2). We also noticed that the short chains subsequently polymerized during electrochemical characterization in MeTriHTFSI electrolyte (Figure S23-S24).

As stated above, we speculate that the rearrangements observed are related to rearrangements of the radical intermediates and the polymerization voltammograms provide some support for this hypothesis (Figure 2c (NQ-EPE) and S25 (QzH2-EPE)). The irreversible anodic peaks correspond to oxidation of neutral trimers to the radical intermediates that ultimately lead to the coupling of trimer segments. With increased MeCN content in the polymerization solution the oxidation peak shifts towards lower potentials, suggesting stabilization of the oxidation products or destabilization of the reactants. As the reactants in all cases are insoluble neutral trimers irrespective of polymerization electrolyte, the latter possibility is unlikely. If the oxidation products, i.e. the charged radical intermediates, become increasingly soluble as the $\mathrm{MeCN}$ content increases, the energy for the oxidation products would be stabilized by the same mechanism. Hence, the shift in peak position could be accounted for by the transient dissolution of the oxidation products (stage ii, Scheme 2). Due 
to the subsequent radical coupling and deprotonation the soluble radical intermediates are prevented from leaving the electrode surface and re-precipitate instead (stage iv, Scheme 2).

In summary, rearrangements of the deposited trimer layer during PDP are required to reach the full charge storage capacity of the materials. Such rearrangement requires transient dissolution of the material. Dissolution of the initial reactants, however, would lead to loss of active materials and hence, rearrangements must be accomplished by intermediate species formed during polymerization instead. A finite solubility of one or more of the reaction intermediates can be accomplished by judicious choice of solvent. We thus propose the polymerization mechanism for PDP that includes 1) oxidation of the neutral trimer, 2) dissolution of radical cations, 3) radical-radical coupling, and 4) re-deposition of oligomers. The obtained maximum capacity from the optimized polymerization solution is close to the theoretical capacity and all the characterized polymers below are obtained under optimized polymerization conditions, namely $0.1 \mathrm{M}$ MeTriHTFSI/MeCN/ $\mathrm{H}_{2} \mathrm{O}(\mathrm{Vol} \mathrm{MeCN}$ : $67 \%$ ) for NQ-EPE and 0.1M MeTriHTFSI/MeCN/H ${ }_{2} \mathrm{O}$ (Vol MeCN: 75\%) for QzH $\mathrm{Q}_{2}-\mathrm{EPE}$.

\subsection{Characterization of individual electrodes}

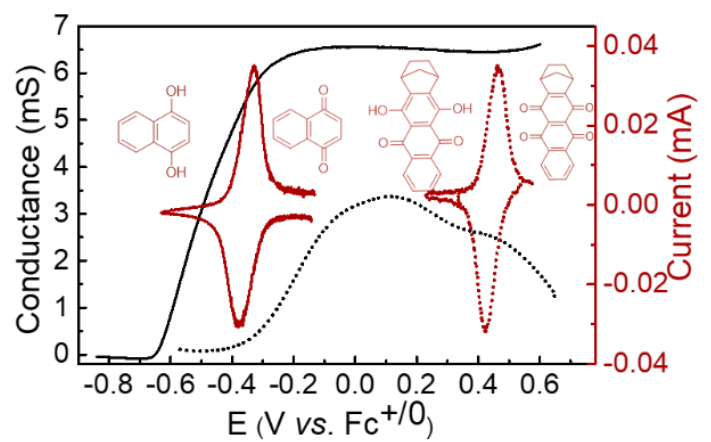

Figure 3. In situ conductance (black) on IDA Au-electrode in 0.1M MeTriHTFSI/MeCN and cyclic voltammograms (red brown) on glassy carbon electrodes in MeTriHTFSI of poly (NQ-EPE) (solid

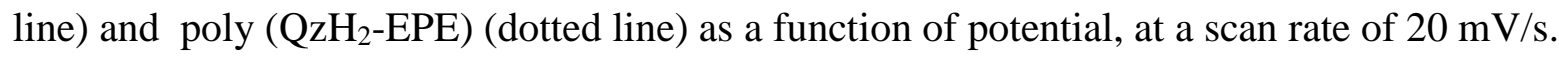

In situ conductance measurements were used to monitor the dependence of polymer conductance on the applied potential. The results show that appreciable conductance is observed at potentials 
above $-0.65 \mathrm{~V}\left(v s . \mathrm{Fc}^{+/ 0}\right)$ and $-0.38 \mathrm{~V}\left(v s . \mathrm{Fc}^{+/ 0}\right)$ for poly (NQ-EPE) and poly $\left(\mathrm{QzH}_{2}-\mathrm{EPE}\right)$, respectively (Figure 3). The increase of conductance is related to the oxidation (or doping) of the polymer backbone: upon oxidation the polymer backbone becomes positively charged and the formed radical cation (polaron) is mobile and acts as charge carrier. With the formation of charge carriers upon oxidation of the polymer backbone, the conductance of the material therefore increases rapidly over a narrow potential region. The conductance of poly (NQ-EPE) reached a rather constant value $(\sim 6 \mathrm{mS})$ at potentials above $-0.2 \mathrm{~V}\left(v s . \mathrm{Fc}^{+/ 0}\right)$. This is a common feature of conducting polymers that is related to the continuous doping over a wide potential region. ${ }^{[40,41]}$ Poly ( ${ }^{2 z H}$-EPE) shows a markedly different dependence of the conductance on potential with a clear maximum of $3.4 \mathrm{mS}$, centered at $0.1 \mathrm{~V}\left(v s . \mathrm{Fc}^{+/ 0}\right)$. Such behavior has more resemblance with redox hopping between redox sites and we hypothesize that this can be traced to the short polymer chains in poly $\left(\mathrm{QzH}_{2}-\mathrm{EPE}\right)$ since shorter chains would give a narrower potential region where the polymer is redox active. Irrespective of transport mechanism, the conductance provided by the polymer backbone was found to be sufficient to allow efficient electron transport pathways for the pendant group redox conversion without using any conducting additives.

Poly (NQ-EPE) and poly $\left(\mathrm{QzH}_{2}\right.$-EPE) show one pair of redox peaks centered at $-0.35 \mathrm{~V}\left(v s . \mathrm{Fc}^{+/ 0}\right)$ and $0.45 \mathrm{~V}\left(v s . \mathrm{Fc}^{+/ 0}\right)$, respectively. The two redox reactions are assigned to the two-electron redox conversion of the respective pendant group that, in both cases, is assumed to be coupled to the transfer of two charge-balancing protons (Scheme 1). The ratios between the anodic and cathodic peaks currents are unity $\left(i_{p(r e d)} / i_{p(o x)}=1\right)$ suggesting that both of the pendant group redox conversions are chemically reversible. Although the anodic and cathodic peak potentials do not coincide even at the lowest scan rate used $(0.1 \mathrm{mV} / \mathrm{s})$, the Gaussian peak shape suggests that the redox reactions appear as surface confined redox processes, indicating full conversion of the layer. As the scan rate was increased the peak separation continuously increased (Figure S28-29) and at a scan rate of around 2 $\mathrm{mV} / \mathrm{s}$ there was a distinct increase in the peak drift with scan rate. The peak drifts indicate that the redox reactions are too slow to keep up with the rate by which the potential is changed, i.e. the rates 
of redox conversion are of the order of $1 \mathrm{~s}^{-1}$. In addition, in NQ-EPE the merged two-electron transfer process splits into two one-electron processes at high scan rates suggesting that the formation of the NQ radical intermediate, in this case, is faster than its consumption. Nevertheless, integration of the redox peaks shows that the total charge is almost constant with scan rate between $2 \mathrm{mV} / \mathrm{s}$ and 20 $\mathrm{mV} / \mathrm{s}$ showing that the pendants are almost fully converted even at a scan rate of $20 \mathrm{mV} / \mathrm{s}$ (insets, Figure S28-29).

Poly $\left(\mathrm{QzH}_{2}\right.$-EPE) exhibited a second pair of redox peaks centered at $-0.4 \mathrm{~V}\left(v_{s} . \mathrm{Fc}^{+/ 0}\right)$ corresponding to the reduction of $\mathrm{Qz}\left(\mathrm{QzH}_{2} / \mathrm{QzH}_{4}\right)($ Figure $\mathrm{S} 30)$. Compared to the $\mathrm{Qz} / \mathrm{QzH}_{2}$ peak the $\mathrm{QzH} / \mathrm{QzH}_{4}$ peak is much smaller which is likely an effect of redox mismatch between the polymer backbone and the $\mathrm{QzH}_{2} / \mathrm{QzH}_{4}$ redox reaction. While the $\mathrm{Qz} / \mathrm{QzH} 2$ redox reaction occurs within the conducting region of poly $\left(\mathrm{QzH}_{2}-\mathrm{EPE}\right)$ the $\mathrm{QzH}_{2} / \mathrm{QzH}_{4}$ redox process falls below the conducting region of the polymer backbone. The redox mismatch for the second reduction leads to an incomplete redox conversion and, hence, a low capacity for the redox reaction (Figure S30). The redox-matched redox process $(\mathrm{Qz} / \mathrm{QzH} 2)$, on the other hand, shows a capacity close to the theoretical capacity ( $\sim 88 \%)$.

In order to confirm the peak assignment above the intensity change of the IR absorption from characteristic carbonyl vibrational peaks upon reduction/oxidation was monitored. Upon oxidation of poly $\left(\mathrm{QzH}_{2}\right.$-EPE) the absorption peak at $1631 \mathrm{~cm}^{-1}$, attributed to the stretching of the Qz-carbonyl group $(-\mathrm{C}=\mathrm{O}),{ }^{[42]}$ strengthened (Figure $\mathrm{S} 27 \mathrm{~b}$ ), indicating that Qz experienced a transition from benzoid structure to quinoid structure as a result of oxidation. In poly (NQ-EPE) the absorption peak at $1627 \mathrm{~cm}^{-1}$, which is assigned to the stretching vibration of the carbonyl group on NQ, weakened upon the reduction (Figure S27a) in agreement with the assignment of the peak to the reduction of NQ.

Individual characterization of the two CRP materials, poly ( $\left.\mathrm{QzH}_{2}-\mathrm{EPE}\right)$ and poly (NQ-EPE), produced by PDP thus shows that both polymers are electrically conductive. The conductance is traced to the EPE backbone and, as for all conducting polymers, ground state conductivity requires 
that the polymer backbone is charged, or doped. Furthermore, in MeTriHTFSI the NQ/NQH 2 redox reaction as well as the $\mathrm{Qz} / \mathrm{QzH}_{2}$ redox conversion is redox-matched with the polymer backbone and the two redox reactions are reversible with capacities close to the theoretical capacity. In contrast, the non-redox matched $\mathrm{QzH}_{2} / \mathrm{QzH}_{4}$ reaction shows capacities far below the theoretical capacity, stressing the importance of redox matching in the CRP design. The dominant part of the material capacity is carried by the redox conversion of the pendant groups occurring at $-0.35 \mathrm{~V}\left(v s . \mathrm{Fc}^{+/ 0}\right)$ for poly (NQ$\mathrm{EPE})$ and $0.45 \mathrm{~V}\left(v s . \mathrm{Fc}^{+/ 0}\right)$ for poly $\left(\mathrm{QzH}_{2}-\mathrm{EPE}\right)$, respectively. Combining the two materials into battery cells with poly (QzH2-EPE) as cathode and poly (NQ-EPE) as anode should hence give a secondary battery with an average voltage output of $0.8 \mathrm{~V}$ and a reversible capacity close to the theoretical capacity of the polymers. We therefore decided to combine the two battery materials into complete battery cells using MeTriHTFSI as electrolyte.

\section{Battery performance}

Batteries with poly (QzH2-EPE) as cathode and poly (NQ-EPE) as anode were assembled as pouch cells with glass microfiber as separator and MeTriHTFSI as electrolyte. Some batteries were fabricated with the cathode as limiting electrode and some with the anode as limiting electrode to enable separate evaluation of the two materials in battery configuration. Both electrodes were predischarged by $\mathrm{CV}$ prior to assembling. As Qz and NQ are the dominant capacity-carrying components they will determine the voltage output $(0.8 \mathrm{~V}$ as suggested from the three-electrode experiments above) as well as the dominant cycling chemistry. During charging, $\mathrm{QzH}_{2}$ is oxidized to $\mathrm{Qz}$ and releases two protons while NQ takes up two protons upon reduction to $\mathrm{NQH}_{2}$ (Scheme 1); the opposite reactions occur during discharge. The dominant process during charge and discharge can thus be described as a proton rocking-chair motion with proton flux towards the anode during charge and opposite flux during discharge. 

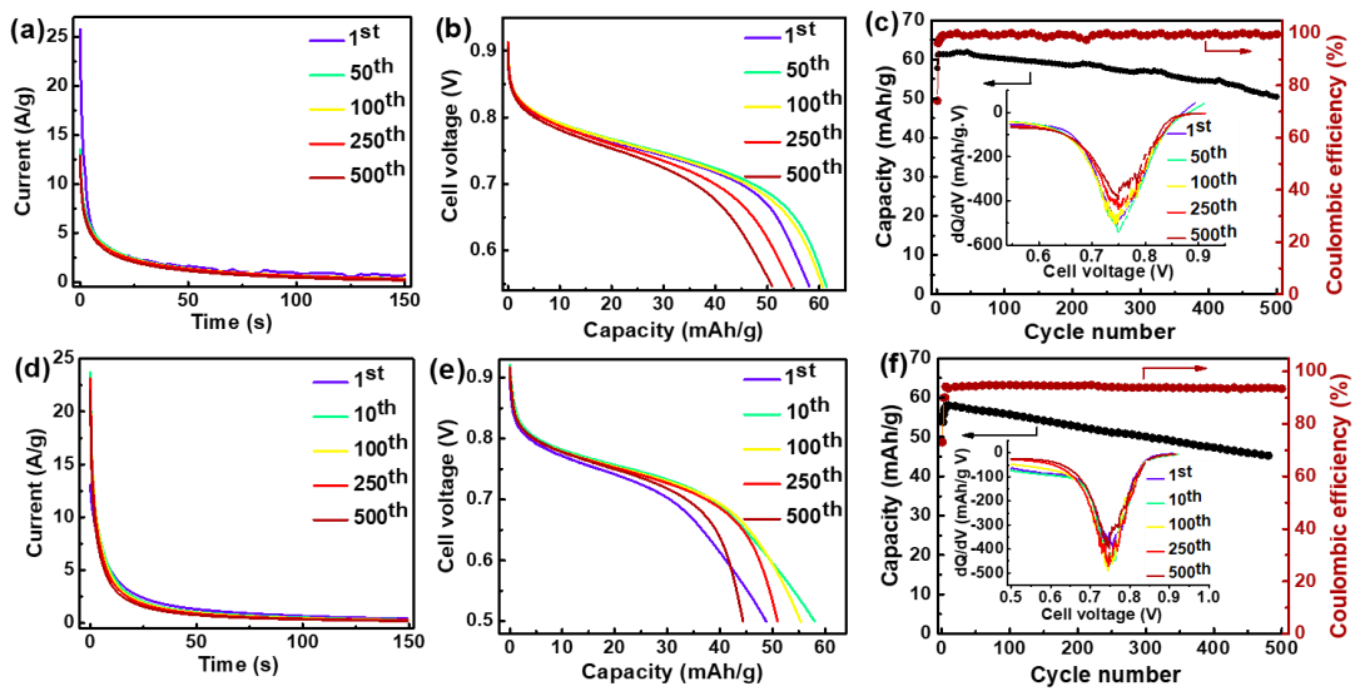

Figure 4. Battery performance using poly (NQ-EPE) as limiting material (upper panels) and poly $\left(\mathrm{QzH}_{2}-\mathrm{EPE}\right)$ as limiting material (bottom panels). Current response as a function of time during the constant voltage charging at $1 \mathrm{~V}(\mathrm{a})$, and $0.95 \mathrm{~V}(\mathrm{~d})$. Discharge profile of different cycles at a current density of $0.3 \mathrm{~A} / \mathrm{g}(\mathrm{b}),(\mathrm{e})$. Corresponding stability and coulombic efficiency (c), (f); the inset figure shows a differential plot of the discharge curve $(\mathrm{dQ} / \mathrm{dV})$ against voltage.

Assembled batteries were charged potentiostatically at $1 \mathrm{~V}$ or $0.95 \mathrm{~V}$, depending on which electrode was capacity-limiting, and discharged galvanostaticially. With poly (NQ-EPE) as limiting material (Figure 4, upper panel) a charging voltage of $1 \mathrm{~V}$ was applied which resulted in an initial charging current of $26 \mathrm{~A} / \mathrm{g}$ which rapidly decayed (Figure 4a). Within $27 \mathrm{~s}$, half of the charging process was completed and full charge was achieved within $150 \mathrm{~s}$ (Figure 4a). In batteries with poly (QzH $2-\mathrm{EPE})$ as limiting material, a slightly lower charging voltage $(0.95 \mathrm{~V})$ was used in order to avoid irreversible oxidation of the electrolyte (Figure S31). In this case the initial current density was $24 \mathrm{~A} / \mathrm{g}$ and half of the charging process was accomplished within $21 \mathrm{~s}$ and charging was completed within $150 \mathrm{~s}$ (Figure 4d).

Galvanostatic discharge of both battery types gave voltage plateaus centered at around $0.8 \mathrm{~V}$ (Figure $4 \mathrm{~b}$ and $4 \mathrm{e}$ ). Distinct peaks in $\mathrm{dQ} / \mathrm{dV}$-plots indicated that the highest capacity was observed at $0.78 \mathrm{~V}$ (insets, Figure $4 \mathrm{c}$ and $4 \mathrm{f}$ ), corresponding well to the difference in formal potential between the 
$\mathrm{NQ} / \mathrm{NQH}_{2}$ and the Qz/QzH 2 redox conversion (Figure 3a). A significant residual capacity below 0.65 $\mathrm{V}$ was also observed in the discharge curve as a result of the backbone doping process. With poly (NQ-EPE) as limiting material the discharge capacity was $62 \mathrm{mAh} / \mathrm{g}$ at a current density of $0.3 \mathrm{~A} / \mathrm{g}$ (4.5 C), which is about $80 \%$ of the theoretical capacity of the NQ/NQH 2 redox conversion $(78 \mathrm{mAh} / \mathrm{g})$. The discharge capacity increased gradually during the first 50 cycles probably as a result of increased swelling of the polymer during cycling. Polymer swelling was supported by EQCM measurements as a continuous mass increase upon cycling in MeTriHTFSI was observed (Figure S33). The anodelimited battery retained $80 \%$ of the highest observed capacity after 500 cycles (Figure 4c) and the peak positions observed in the $\mathrm{dQ} / \mathrm{dV}$-plot were well-preserved, indicating that the non-limiting cathode was sufficiently stable as to provide a stable reference potential. The close to $100 \%$ coulombic efficiency also suggests that no (or only minor) irreversible side reactions occurred in the anode-limited device. This $0.8 \mathrm{~V}$ battery was also proved to be able to power a red light-emitting diode by utilizing three batteries in series (Figure S34). The cathode-limiting device showed a similar initial discharge capacity $(58 \mathrm{mAh} / \mathrm{g})$ as the anode-limited device at a current density of $0.3 \mathrm{~A} / \mathrm{g}(4.5$ C), corresponding to $85 \%$ of the theoretical capacity of the Qz/QzH 2 redox conversion $(68 \mathrm{mAh} / \mathrm{g})$. The coulombic efficiency was, however, much lower $(\sim 95 \%)$ and $75 \%$ of the capacity was retained after 500 cycles.
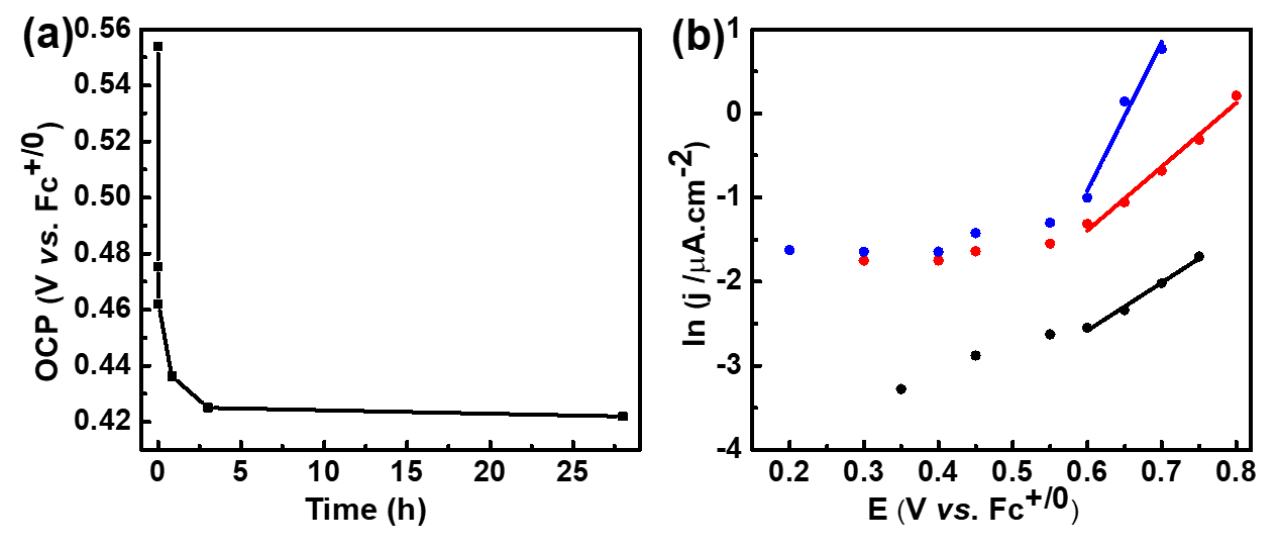

Figure 5. (a) Open circuit potential relaxation of poly $\left(\mathrm{QzH}_{2}-\mathrm{EPE}\right)$ in a three-electrode setup after potentiostatic charging at $0.7 \mathrm{~V}$ for $150 \mathrm{~s}$. (b) Plots of leakage current of bare glassy carbon (black), 
poly (EPE) on glassy carbon (red), and poly (QzH 2 -EPE) on glassy carbon (blue) as a function of applied potential in MeTriHTFSI.

In order to investigate the origin of the low coulombic efficiency of the cathode-limited device the self-discharge of the charged cathode was investigated. The poly $\left(\mathrm{QzH}_{2}-\mathrm{EPE}\right)$ electrode was first charged by applying a constant potential of $0.7 \mathrm{~V}\left(v s . \mathrm{Fc}^{+/ 0}\right)$ for $150 \mathrm{~s}$ in a three-electrode setup. The open circuit potential (OCP) was then monitored during a fixed period of time after which the electrode was galvanostatically discharged, the remaining capacity was recorded and the electrode was then recharged and the cycle was repeated. Figure 5a shows that the OCP decayed to $0.43 \mathrm{~V}$ ( $v s$. $\mathrm{Fc}^{+/ 0}$ ) within $3 \mathrm{~h}$ and $33 \%$ of the capacity was lost (Figure S35). During the next $25 \mathrm{~h}$ the OCP slowly dropped to $0.42 \mathrm{~V}\left(v s . \mathrm{Fc}^{+/ 0}\right)$ and an additional loss in capacity of $13 \%$ was observed (Figure S35). In an attempt to determine the mechanism of charge loss in the cathode, leakage current experiments were conducted on poly ( $\mathrm{QzH}_{2}$-EPE) and poly (EPE) covered electrodes, as well as a bare glassy carbon electrode used as reference system. A fixed potential was applied to the electrode and the residual current was measured at various potentials (Figure S36). In all cases the leakage current increased exponentially with potential above $0.6 \mathrm{~V}\left(v s . \mathrm{Fc}^{+/ 0}\right)$, suggesting that the leakage current in all cases was kinetically limited by a redox reaction. The bare electrode also showed an exponential dependence of the leakage current on potential, suggesting that the electrolyte MeTriHTFSI undergoes an irreversible oxidation that, in principle, could partially account for the charge losses. However, the leakage currents observed using polymer-covered electrodes are much higher than that observed with the bare glassy carbon, which could be due to the larger surface area in these electrodes covered by polymer. However, it is unlikely that the increased surface area could account for the order-of-magnitude increase in leakage current. A more likely explanation is that irreversible oxidation reaction of the polymer dominates the current leakage. As the poly $\left(\mathrm{QzH}_{2}-\mathrm{EPE}\right)-$ and the poly (EPE)-covered electrodes show similar responses it is likely that the reaction is related to the polymer backbone rather than the pendant. Over-oxidation is a feature that has been observed for conducting polymers that could be the origin of the leakage current, ${ }^{[43-46]}$ but we cannot exclude the 
possibility that the polymer-centered redox reaction is triggered by the degradation of the electrolyte at this stage. We thus conclude that the low coulombic efficiency in the cathode-limited cell is due to irreversible redox reactions related to the polymer backbone. The strong dependence of this reaction on potential makes it possible to suppress the charge loss and improve the coulombic efficiency by avoiding complete oxidation of the cathode, as was demonstrated in the anode-limiting device.

\section{Conclusion}

In this report, we have successfully produced polymer films by PDP using trimeric, thiophene-based starting materials with attached pendant quinone functional groups. The use of trimers allows for polymerization under mild conditions and the PDP method allows for $100 \%$ utilization of the starting materials. We have shown that successful PDP relies on the transient dissolution and rearrangement of the intermediate radical cation state during polymerization that can be controlled by the solvent composition of the polymerization electrolyte. The choice of polymerization solvent composition can tune the polymer properties and it strongly affects polymer morphologies, polymer lengths and the electrochemical properties of the resulting polymer. Polymers formed using optimized PDP conditions showed appreciable conductance and reversible, fast redox conversion of the quinone pendants with capacities close to the theoretical capacity. The polymer conductance enabled use of the materials as active electrode materials directly, without the need for any conducting additives. By utilizing two different pendants, NQ and Qz, a potential difference of $0.8 \mathrm{~V}$ was achieved in protic ionic liquid electrolyte. By combining the two materials an all-organic proton rocking-chair battery with protic ionic liquid electrolyte was produced and tested.

\section{Experimental}

All solvents and chemicals were purchased from Sigma Aldrich and were used without further purification. The MeTri-based ionic liquid electrolyte was prepared through the protonation of MeTri by bis(trifluoromethylsulfonyl)imide (HTFSI) in a glove box as described in a previous study. ${ }^{[25]}$ Herein $30 \%$ degree of protonation was used due to the high conductivity $\left(2 \cdot 10^{-2} \mathrm{~S} \mathrm{~cm}^{-1}\right.$ at $\left.25^{\circ} \mathrm{C}\right)$. In 
the resulting MeTriHTFSI ionic liquid electrolyte, 30\% of the MeTri moieties are protonated and act as proton donors $\left(\mathrm{MeTriH}^{+}\right)$while $70 \%$ are in the initial, deprotonated state (MeTri) and serve as proton acceptors. The structure of $\mathrm{MeTriH}^{+}$and MeTri are shown in Scheme 1. The TFSI anion, on the other hand, acts as charge-compensating ion for the positively charged polymer backbone during the doping process that renders the polymer conducting.

5.1. Synthesis: The 3,4-ethylenedioxythiophene (EDOT)-3,4-propylenedioxythiophene (ProDOT)-EDOT (EPE) trimeric precursor was synthesized as previously reported. ${ }^{17}$ The synthetic route to attach NQ and Qz onto EPE is shown in Figure S1. A fused norbornane is used to improve solubility of the Qz unit and to stabilize the oxidized species similar to the strategy employed by Aziz and co-workers. ${ }^{19}$ Supporting information Section S1 gives full details regarding the synthesis and characterization of these polymer precursors. Structural characterization using ${ }^{1} \mathrm{H}-\mathrm{NMR}$ and ${ }^{13} \mathrm{C}$ NMR are shown in Figure S2-S13.

5.2. Post-deposition polymerization (PDP): $\mathrm{QzH}_{2}-\mathrm{EPE}$ and NQ-EPE trimers were dissolved in N-methyl-2-pyrrolidone (NMP) solvent which contains $10 \mathrm{mg} / \mathrm{mL}$ polyvinylidene fluoride (PVDF), to make a $100 \mathrm{mg} / \mathrm{mL}$ trimer solution. The mass-ratio of trimer and PVDF binder was set to be 10:1. The trimer solution was drop-cast onto a glassy carbon plate (HTW, Germany, $40 \mathrm{~mm}$ x $8 \mathrm{~mm}$ x 2 $\mathrm{mm}$ ) that was used as current collector, followed by vacuum drying at 1 bar at $25^{\circ} \mathrm{C}$ for 10 minutes to remove the solvent. The resulting glassy carbon plate was then immersed into $0.1 \mathrm{M}$ MeTriHTFSI/MeCN/H $\mathrm{H}_{2} \mathrm{O}$ solution.

Cyclic voltammetry (CV) was then used to electro-polymerize the trimer layer in a three-electrode setup using an Autolab PGSTAT302N potentiostat (Ecochemie, The Netherlands). The CV polymerization was conducted at a scan rate of $20 \mathrm{mV} / \mathrm{s}$ between -0.1 and $0.6 \mathrm{~V}$ for 6 scans in $0.1 \mathrm{M}$ MeTriHTFSI/MeCN/H${ }_{2} \mathrm{O}$. A series of solvent mixtures with different $\mathrm{MeCN}$ volume fraction, 0\%, $25 \%, 33 \%, 50 \%, 67 \%, 75 \%$ and $100 \%$, were used. In 0.1M MeTriHTFSI/MeCN/ $\mathrm{H}_{2} \mathrm{O}$ solution NQEPE starts to dissolve when the MeCN volume fraction is higher than 67\% while $\mathrm{QzH}_{2}-\mathrm{EPE}$ starts to 
dissolve when the $\mathrm{MeCN}$ volume fraction is higher than $75 \%$. The glassy carbon working electrode was connected to the outer circuit as shown in Figure S14. A Pt wire was used as a counter electrode and the reference electrode consisted of an $\mathrm{Ag}$ wire immersed into a $\mathrm{MeCN}$ solution containing $0.1 \mathrm{M}$ MeTriHTFSI and 0.01 $\mathrm{M} \mathrm{AgNO}_{3}$, which was kept in a separate compartment with a porous glass frit. The reference electrode was calibrated against ferrocene after each set of experiments, and all potentials are reported against the ferrocene formal potential $\left(\mathrm{Fc}^{+/ 0}\right)$. All polymerizations were conducted in $\mathrm{N}_{2}$ purged solutions and a $\mathrm{N}_{2}$ pressure over the electrolyte was sustained throughout the experiment. After polymerization, the polymer-covered glassy carbon electrode was taken out from the electrolyte and was allowed to dry at ambient conditions for 30 minutes. Thick polymer films suffered from cracking after solvent evaporation, limiting the maximum mass loading to $2 \mathrm{mg} / \mathrm{cm}^{2}$.

5.3. Electrochemical characterization: For general electrochemical characterizations, a low polymer mass loading was used. $1.0 \mu \mathrm{L}$ of the above $100 \mathrm{mg} / \mathrm{mL}$ trimer solution, corresponding to $0.1 \mathrm{mg}$ dissolved trimer was drop-cast onto glassy carbon with the area of around $0.1 \mathrm{~cm}^{2}$, corresponding to $1 \mathrm{mg} / \mathrm{cm}^{2}$. After PDP the resulting polymer was characterized in a three-electrode setup in MeTriHTFSI electrolyte using an Autolab PGSTAT302N potentiostat (Ecochemie, The Netherlands) in a $\mathrm{N}_{2}$-filled glovebox. The reference electrode consisted of an $\mathrm{Ag}$ wire immersed in

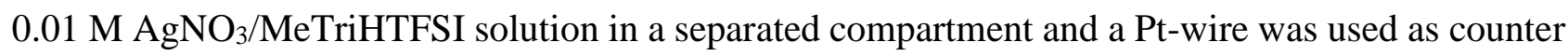
electrode.

For in situ characterizations, a $10 \mathrm{mg} / \mathrm{mL}$ trimer solution excluding PVDF was used. For in situ conductance measurement, the working electrode was an interdigitated array (IDA) electrode with 90 pairs of gold bands on a glass substrate $(10 \mu \mathrm{m}$ between bands, $150 \mathrm{~nm}$ high, MicruX Technologies, Spain). In situ conductance measurements were conducted using an Autolab PGSTAT302N potentiostat (Ecochemie, The Netherlands) equipped with a bipotentiostat module using CV. A potential bias of $1 \mathrm{mV}$ was applied between the two working electrodes. ${ }^{[30]}$ The polymer conductance was investigated in $0.1 \mathrm{M}$ MeTriHTFSI/MeCN electrolyte. $\mathrm{N}_{2}$ was used to purge the electrolyte 
solution and a $\mathrm{N}_{2}$ pressure over the electrolyte was sustained throughout the experiment. For in situ electrochemical Quartz Crystal Microbalance (EQCM) measurements, the working electrode was gold coated AT-cut quartz EQCM-crystals $(8.95 \mathrm{MHz}-30 \mathrm{kHz}, \emptyset 5 \mathrm{~mm})$. The PDP was conducted using CV on a VersaSTAT potentiostat (VersaSTAT 4, AMETEK) while the frequency change of the EQCM crystal during CV measurement was recorded. The frequency change was subsequently converted to a mass change using the Sauerbrey equation. ${ }^{[31]}$

5.4. Battery evaluation: For battery evaluations, $5.0 \mu \mathrm{L}$ of the above $100 \mathrm{mg} / \mathrm{mL}$ trimer solution with $10 \mathrm{mg} / \mathrm{mL}$ PVDF, corresponding to $0.5 \mathrm{mg}$ dissolved trimer was drop-cast onto a glassy carbon yielding a covered area of $0.25-0.35 \mathrm{~cm}^{2}$, with a corresponding mass loading of 1.4-2.0 $\mathrm{mg} / \mathrm{cm}^{2}$. The prepared poly $\left(\mathrm{QzH}_{2}-\mathrm{EPE}\right)$ and poly (NQ-EPE) electrodes were assembled into a pouch cell in a $\mathrm{N}_{2}$-filled glove box. A glass microfiber filter (CAT NO. 1820-055, Whatman) was used as separator and MeTriHTFSI was used as electrolyte. In order to evaluate the properties of the two polymers separately, two types of cells were prepared; one was capacity-limited by the poly (NQ$\mathrm{EPE})$ anode and the other one by the poly $\left(\mathrm{QzH}_{2}-\mathrm{EPE}\right)$ cathode. The mass-ratio of active materials at the two electrodes was 1:4. In the anode-limiting case the total theoretical capacity of cathode side is 3.5 times of the anode while in the cathode-limiting case the total theoretical capacity of anode side is 4.6 times of the cathode. The electrodes were pre-discharged by CV before they were assembled into a battery. The battery performance was investigated using an Autolab PGSTAT302N potentiostat.

5.5. Other characterization: Scanning Electron Microscopy (SEM) was carried out using a Leo Gemini 1550 FEG SEM instrument (Zeiss, Germany) equipped with both an in-lens and EDX detector. The sample was pre-coated with a conductive gold layer prior to imaging.

\section{Supporting Information}

Supporting Information is available from the Wiley Online Library or from the author

\section{Acknowledgements}


This work was funded by the Swedish Energy Agency and the Research Council Formas. Huan Wang thank the China Scholarship Council (CSC) for a PhD study scholarship.

\section{Conflict of Interests}

The authors declare no competing financial interest

\section{Keywords}

proton battery, organic battery, conducting redox polymers, quinone, PEDOT, polymerization

\section{References}

[1] P. Poizot, F. Dolhem, Energy Environ. Sci. 2011, 4, 2003.

[2] X.-P. Gao, H.-X. Yang, Energy Environ. Sci. 2010, 3, 174.

[3] M. Armand, J.-M. Tarascon, Nature 2008, 451, 652.

[4] D. Larcher, J.-M. Tarascon, Nature Chem 2015, 7, 19.

[5] K. Oka, C. Strietzel, R. Emanuelsson, H. Nishide, K. Oyaizu, M. Strømme, M. Sjödin, Electrochemistry Communications 2019, 105, 106489.

[6] Q. Zhao, W. Huang, Z. Luo, L. Liu, Y. Lu, Y. Li, L. Li, J. Hu, H. Ma, J. Chen, Sci. Adv. 2018, 4, eaao1761.

[7] M. Lécuyer, J. Gaubicher, A.-L. Barrès, F. Dolhem, M. Deschamps, D. Guyomard, P. Poizot, Electrochemistry Communications 2015, 55, 22.

[8] S. Er, C. Suh, M. P. Marshak, A. Aspuru-Guzik, Chem. Sci. 2015, 6, 885.

[9] M. Namazian, S. Siahrostami, M. L. Coote, Journal of Fluorine Chemistry 2008, 129, 222.

[10] R. R. S. Shi, M. E. Tessensohn, S. J. L. Lauw, N. A. B. Y. Foo, R. D. Webster, Chem. Commun. 2019, 55, 2277.

[11] W. Yin, A. Grimaud, I. Azcarate, C. Yang, J.-M. Tarascon, J. Phys. Chem. C 2018, 122, 6546.

[12] R. Emanuelsson, M. Sterby, M. Strømme, M. Sjödin, J. Am. Chem. Soc. 2017, 139, 4828.

[13] N. Gupta, H. Linschitz, J. Am. Chem. Soc. 1997, 119, 6384.

[14] M. Quan, D. Sanchez, M. F. Wasylkiw, D. K. Smith, J. Am. Chem. Soc. 2007, 129, 12847. 
[15] Y. Hui, E. L. K. Chng, C. Y. L. Chng, H. L. Poh, R. D. Webster, J. Am. Chem. Soc. 2009, 131, 1523.

[16] Y. Liang, Y. Jing, S. Gheytani, K.-Y. Lee, P. Liu, A. Facchetti, Y. Yao, Nature Mater 2017, 16, 841.

[17] C. Strietzel, M. Sterby, H. Huang, M. Strømme, R. Emanuelsson, M. Sjödin, Angew. Chem. Int. Ed. 2020, 59, 9631.

[18] K. Nueangnoraj, T. Tomai, H. Nishihara, T. Kyotani, I. Honma, Carbon 2016, 107, 831.

[19] L. Tong, Y. Jing, R. G. Gordon, M. J. Aziz, ACS Appl. Energy Mater. 2019, 2, 4016.

[20] D. P. Tabor, R. Gomez-Bombarelli, L. Tong, M. J. Aziz, A. Aspuru-Guzik, 22. https://doi.org/10.26434/chemrxiv.6990053.v1

[21] L. Hoober-Burkhardt, S. Krishnamoorthy, B. Yang, A. Murali, A. Nirmalchandar, G. K. S. Prakash, S. R. Narayanan, J. Electrochem. Soc. 2017, 164, A600.

[22] B. Yang, L. Hoober-Burkhardt, S. Krishnamoorthy, A. Murali, G. K. S. Prakash, S. R. Narayanan, J. Electrochem. Soc. 2016, 163, A1442.

[23] K. Wedege, E. Dražević, D. Konya, A. Bentien, Sci Rep 2016, 6, 39101.

[24] R. Emanuelsson, H. Huang, A. Gogoll, M. Strømme, M. Sjödin, J. Phys. Chem. C 2016, 120, 21178.

[25] C. Karlsson, C. Strietzel, H. Huang, M. Sjödin, P. Jannasch, ACS Appl. Energy Mater. 2018, 1, 6451.

[26] L. Timperman, A. Vigeant, M. Anouti, Electrochimica Acta 2015, 155, 164.

[27] U. A. Rana, M. Forsyth, D. R. MacFarlane, J. M. Pringle, Electrochimica Acta 2012, 84, 213.

[28] M. Anouti, L. Timperman, Phys. Chem. Chem. Phys. 2013, 15, 6539.

[29] H. Wang, R. Emanuelsson, A. Banerjee, R. Ahuja, M. Strømme, M. Sjödin, J. Phys. Chem. C 2020, $124,13609$.

[30] C. Karlsson, H. Huang, M. Strømme, A. Gogoll, M. Sjödin, Electrochimica Acta 2015, 179, 336. 
[31] M. Sterby, R. Emanuelsson, X. Huang, A. Gogoll, M. Strømme, M. Sjödin, Electrochimica Acta $2017,235,356$.

[32] H. Wang, R. Emanuelsson, H. Liu, K. Edström, F. Mamedov, M. Strømme, M. Sjödin, ACS Appl. Energy Mater. 2019, 2, 7162.

[33] C. D. Spicer, M. A. Booth, D. Mawad, A. Armgarth, C. B. Nielsen, M. M. Stevens, Chem 2017, 2, 125.

[34] T. Takano, H. Masunaga, A. Fujiwara, H. Okuzaki, T. Sasaki, Macromolecules 2012, 45, 3859.

[35] W. Baik, W. Luan, R. H. Zhao, S. Koo, K.-S. Kim, Synthetic Metals 2009, 159, 1244.

[36] T. M. Swager, Macromolecules 2017, 50, 4867.

[37] H. J. Ahonen, J. Lukkari, J. Kankare, Macromolecules 2000, 33, 6787.

[38] G. Zotti, S. Zecchin, G. Schiavon, L. “Bert” Groenendaal, Chem. Mater. 2000, 12, 2996.

[39] Y. Seki, M. Takahashi, M. Takashiri, RSC Adv. 2019, 9, 15957.

[40] M. Wieland, C. Malacrida, Q. Yu, C. Schlewitz, L. Scapinello, A. Penoni, S. Ludwigs, Flex. Print. Electron. 2020, 5, 014016.

[41] G. Salinas, J.-A. Del-Oso, P.-J. Espinoza-Montero, J. Heinze, B. A. Frontana-Uribe, Synthetic Metals 2018, 245, 135.

[42] V. Rajshekar Shetty, S. S. Gurukar, R. Marriappa, M. M. Kittappa, D. H. Nagaraju, New J. Chem. $2015,39,8534$.

[43] H. Olsson, M. Sjödin, E. B. Jämstorp, M. Strømme, L. Nyholm, Green 2014, 4.

[44] H. Olsson, E. B. Jämstorp, M. Strømme, M. Sjödin, Electrochemistry Communications 2015, 50, 43.

[45] H. Olsson, M. Strømme, L. Nyholm, M. Sjödin, J. Phys. Chem. C 2014, 118, 29643.

[46] A. Zykwinska, W. Domagala, B. Pilawa, M. Lapkowski, Electrochimica Acta 2005, 50, 1625. 